\title{
PERMISSIVE LEFT-TURN BEHAVIOR AT THE FLASHING YELLOW ARROW IN THE PRESENCE OF PEDESTRIANS
}

\author{
Patrick Marnell ${ }^{1}$, Halston Tuss ${ }^{1}$, David Hurwitz ${ }^{1}$, Kirk Paulsen ${ }^{2}$ \& Chris Monsere $^{2}$ \\ ${ }^{1}$ Civil \& Construction Engineering, Oregon State University, Corvallis, Oregon, USA \\ ${ }^{2}$ Civil and Environmental Engineering, Portland State University, Portland, Oregon, USA \\ Email: marnellp@gmail.com
}

\begin{abstract}
Summary: Use of the flashing yellow arrow indication for permissive left-turn control has become more common in the U.S. since it was adopted in the 2009 Edition of the Manual on Uniform Traffic Control Devices. A complete understanding of the safety implications at signalized intersections is critically important. This paper examines the results of a permissive left-turn driver behavior study conducted in a high fidelity driving simulator. The experimental results suggest 1) that when there are more pedestrians present in the conflicting crosswalk, the driver's average fixation duration on crossing pedestrians is greater than when there is minimal pedestrian activity; 2) that $4 \%$ to $7 \%$ of drivers do not fixate on pedestrians in the crosswalk when completing their left turn; and 3) that $39 \%$ of drivers do not fixate on likely pedestrian locations when pedestrians are not present.
\end{abstract}

\section{INTRODUCTION}

A driver facing a permissive left-turn traffic signal indication must yield the right-of-way to opposing traffic (vehicles and bicycles) and conflicting pedestrians in the crosswalk. In many situations the driver workload is elevated and drivers fail to scan for pedestrians while performing permissive left-turns (Lord et al., 1998). This is particularly an issue in suburban settings where driver expectation of pedestrians is low. The National Safety Council describes inattention as "cognitive distraction" which "contributes to a withdrawal of attention from the visual scene, where all the information the driver sees is not processed" (National Safety Council, 2010). More simply, inattention occurs when a driver is looking directly at something and does not detect the details of the object due to a mental processing conflict.

Past research has demonstrated the flashing yellow arrow (FYA) as the preferred signal indication for protected/permissive left-turn (PPLT) based on driver comprehension. In 2003, NCHRP Report 493, "Evaluation of Traffic Signal Displays for Protected/Permissive Left-Turn Control," performed a comprehensive evaluation of PPLT alternatives and arrived at the recommendation to include the FYA in future editions of the MUTCD (Brehmer et al., 2003). The MUTCD now includes guidelines for FYA operation (2009). In a follow-up study in 2005, Knodler and Noyce used eye tracking equipment in a driving simulator to investigate driver glance patterns and fixations. Eleven subjects drove a total of 66 simulated intersection interactions. Knodler and Noyce found that 90 percent of the drivers initially focused on the PPLT signal head before focusing on the opposing through traffic to find an adequate gap (2005). Finally, Knodler et al. demonstrated that a driving simulator is an appropriate tool to conduct driver comprehension experiments of PPLT signal controls (2001). In their study, they 
compared the analysis of the results of 211 subjects simulator trials with a static paper based survey and found more realistic results in the simulator.

The Oregon Department of Transportation (ODOT) was an early adopter and a national leader in the application of the FYA indication for PPLT signal operation-requiring installation of the FYA on all state highways operating PPLT phasing (ODOT, 2006). Other jurisdictions in Oregon have also adopted a similar policy. Washington County, Oregon has taken this further, replacing many intersections that had protected left turn operations with FYA PPLT operation. Due to reports of a high number of conflicts between pedestrians and permissive left-turning vehicles, operation of the FYA was halted at several signalized intersections in Washington County, Oregon. These intersections provided the research with motivation and context to study the interaction between pedestrians and drivers making permissive left-turn maneuvers.

This research investigated the influence of three factors related to the permissive left-turn vehicle conflict with pedestrians: opposing traffic volumes, pedestrian volumes and direction, and signal display configurations. This paper presents a subset of results from the study related to the influence of pedestrian volumes and direction. Using a high fidelity driving simulator, the research sought to further our understanding of the fundamental factors of the permissive leftturning vehicle conflict with pedestrians.

The remainder of the paper is organized as follows: the research apparatus, method and protocol are described in the following section, followed by the presentation of results and conclusions.

\section{METHODOLOGY}

This research was conducted in a high fidelity driving simulator. To build the environment, candidate FYA locations were identified from historical crash data from installations in Washington County, Oregon. From this list, a selected set of intersections was identified and elements of those intersections (approach widths, lane configurations, signal head configurations, and adjacent land use) were modeled in the OSU Driving Simulator. Drivers completed 24 independent left-turn maneuvers during a 45 minute experimental trial.

\section{OSU Driving Simulator Description}

The OSU Driving Simulator is a high-fidelity motion base simulator providing approximately 220 degrees of projection on three forward projection screens, one rear screen, and two LCD screens on the side view mirrors (Figure 1). Three liquid crystal silicon projectors, with resolution of $1400 \times 1050$, are used to project the $180^{\circ} \times 40^{\circ}$ front view. A digital light processing projector is used to display a rear image for the driver's center mirror.

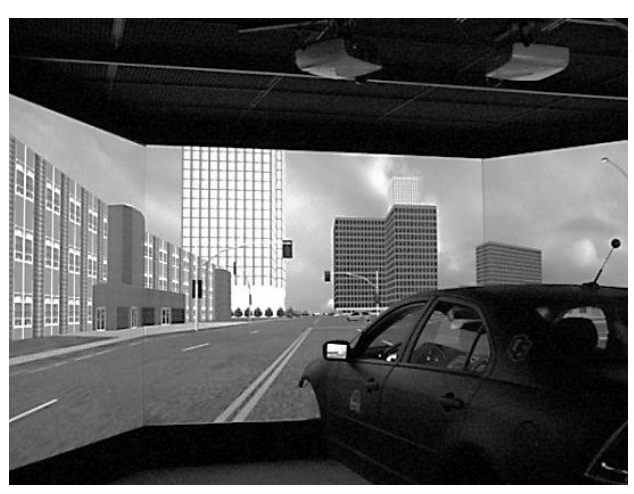

Figure 1. OSU Driving Simulator

The simulator consists of a full 2009 Ford Fusion cab mounted on top of an electric pitch motion system capable of rotating +/- 4 degrees. The vehicle cab is mounted on the pitch motion system with the driver's eye-point located at the center of the viewing volume. The pitch motion system 
allows for the accurate representation of acceleration or deceleration events. The ambient road sounds are modeled with a surround sound system, as well as sound internal to the vehicle (Oregon State University, 2011).

\section{Eye Tracking Data}

Eye tracking data was collected using a Mobile Eye-XG platform from Applied Science Laboratories (Figure 2). The advanced Mobile Eye-XG allows the user to not only have unconstrained eye movement but also unconstrained head movement, generating a sampling rate of $30 \mathrm{~Hz}$ and an accuracy of 0.5 to 1.0 degree. The subject's gaze is

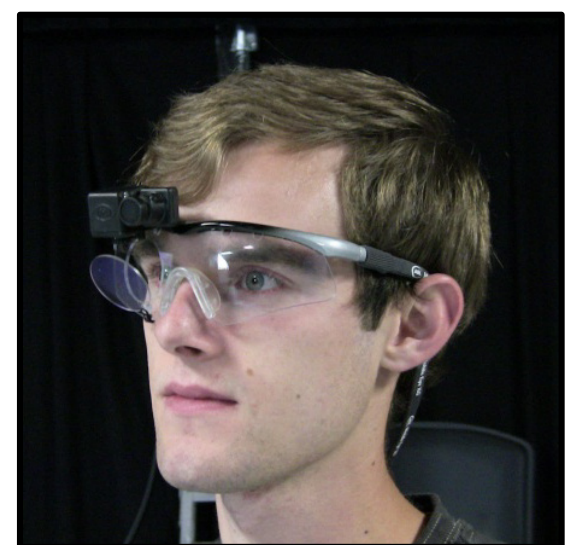

Figure 2. Mobile Eye XG calculated based on the correlation between the subject's pupil position and the reflection of three infrared lights on the surface of the eye. Eye movement consists of fixations and saccades where fixations are points that are focused for more than 100 milliseconds and saccades are when the eye moves to another fixation point.

\section{Subject Recruitment and Sample Size}

Participants in this study were recruited from OSU students and the surrounding community. Participants were required to possess a valid driver's license, not have vision problems, and be physically and mentally capable of legally operating a vehicle. Participants also needed to be deemed competent to provide written informed consent. This study targeted an enrollment of 30 participants with a balance of gender. Researchers did not screen interested participants based on gender until the quota for either males or females had been reached, at which point only the gender with the unmet quota was allowed to participate. In total, 38 drivers participated in the test. There was an over-representation of college aged students - the mean age of subjects was 25.8. Due to simulator sickness 8 drivers did not complete the experiment and data collection errors rendered 3 subject's data unusable. Data was processed and analyzed for the remaining 27 subjects (14 male and 13 female).

\section{Scenario Layout and Intersection Control}

Simulator software, including Internet Scene Assembler, Simcreator, and Google Sketchup were used to create a virtual environment that could be projected around the driver. In an effort to reduce the chances of simulator sickness, the driving scenario was split into four trials of six intersections each. This allowed the subjects the opportunity to take small breaks between trials instead of requiring them to maneuver through all 24 intersections in a single trial. It also allowed for the researchers to introduce one distractor question between each trial. The distractor questions included:

- Did you find that the posted speed limit was appropriate for the road driven?

- How did the presence of bike lanes affect your driving behavior?

- What are your thoughts on the digital dashboard configuration? 
Subjects were directed to conduct a total of six left-turn movements in each trial. Experimental variables included the FYA configuration (3 section dual-arrow or 4 section), combinations of crossing pedestrians, and opposing vehicular volume. In an effort to control bias, the order of the presentation of experimental variables was randomized during the design of the four trails. Additionally, the order in which the four trials were presented to the subjects was randomized. In total, 24 different combinations of these variables were presented to the driver when approaching the intersections.

Tangent sections between intersections measure approximately $1650 \mathrm{ft}$, with $12 \mathrm{ft}$ lanes and $4 \mathrm{ft}$ bike lanes. All intersection approaches consisted of five lanes, two through lanes in each direction and an exclusive left-turn bay. The intersection approaches had a posted speed limit of $45 \mathrm{mph}$.

\section{RESULTS}

The 27 subjects navigating through 24 intersections could produce a maximum of 648 observations. However, due to data collection errors with the eye tracker only 530 observations were collected.

\section{AOI Fixations by Pedestrian Direction}

For the first set of statistical analyses, areas of interest (AOI) were defined. These AOI included: pedestrian, pedestrian area, opposing vehicle, FYA
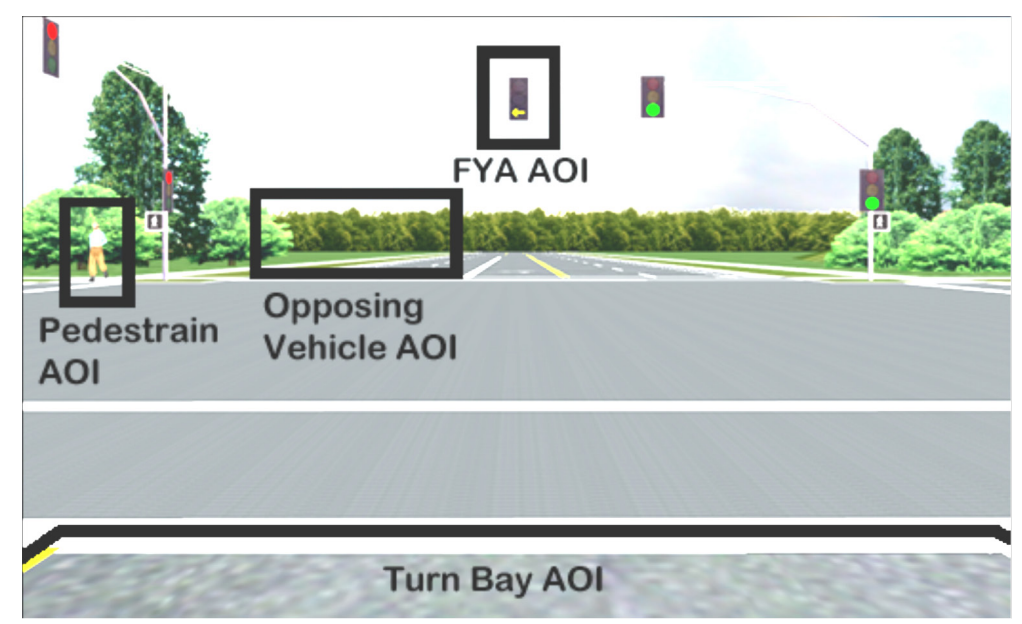

Figure 3. Example areas of interest (AOI) signal head, and turn bay. The Mobile Eye-XG software then calculated fixation durations in these AOI (Figure 3). These data were split by the three pedestrian levels described by the first null hypothesis:

$\boldsymbol{H}_{0}:$ There is no difference in the mean total duration of driver fixations during permitted left-turn maneuvers at signalized intersections operating the FYA with pedestrians walking towards, away, or from both sides.

This resulted in three groups consisting of six experimental scenarios each, which allowed researchers to isolate the impact of individual variable levels. R Statistical Software (2012) was used to perform an ANOVA analysis on the average total fixation durations (ATFD) by cases of pedestrians walking towards, away, or from both sides. Using Tukey's Honest Significant Difference test, 95\% family-wise confidence levels were calculated. Table 1 presents the results of these analyses.

The ANOVA analysis shows that only the fixations on the pedestrians showed any significant differences $(\mathrm{p}<0.001)$. The family-wise comparison shows that between the Toward and Away cases, no statistically significant differences were found. This suggests that fixation durations do not change depending on the direction a single pedestrian is walking in the crosswalk. Recall that 
the only vehicle-pedestrian conflict being tested is that which occurs during a permitted left-turn. It was found that the ATFD for the pedestrian AOI was statistically different when there was a single pedestrian walking toward the subject (Toward) versus four pedestrians (two from each side) (Both). This result was also found between the case of Away vs. Both independent variables. No other significant differences were found $(\alpha=0.05)$.

Table 1. Driver fixations on AOI by pedestrian direction

\begin{tabular}{|l|c|c|c|c|c|c|c|c|c|c|}
\hline \multirow{2}{*}{$\begin{array}{c}\text { Areas of } \\
\text { Interest }\end{array}$} & \multicolumn{3}{|c|}{$\begin{array}{c}\text { Pedestrian direction } \\
\text { of travel }\end{array}$} & ANOVA & \multicolumn{3}{c|}{$\begin{array}{l}\text { Tukey's Honest Significant Differences multiple } \\
\text { comparisons of means with 95\% family-wise } \\
\text { confidence level for ped cases }\end{array}$} \\
\cline { 2 - 12 } & Toward & Away & Both & All & $\begin{array}{c}\text { Toward } \\
\text { vs } \text { Away }\end{array}$ & \multicolumn{2}{c|}{$\begin{array}{c}\text { Toward } \\
\text { vs Both }\end{array}$} & \multicolumn{3}{c|}{$\begin{array}{c}\text { Away } \\
\text { vs Both }\end{array}$} \\
\cline { 2 - 12 } & \multicolumn{3}{|c|}{ ATFD (sec) } & p-value & p-value & Sig & p-value & Sig & p-value & Sig \\
\hline Pedestrians & 1.504 & 1.639 & 2.974 & $<0.001$ & 0.489 & No & $<0.001$ & $\underline{\text { Yes }}$ & $<0.001$ & $\underline{\text { Yes }}$ \\
\hline FYA Signal & 1.730 & 1.783 & 1.625 & 0.704 & 0.958 & No & 0.848 & No & 0.689 & No \\
\hline $\begin{array}{l}\text { Opposing } \\
\text { Vehicles }\end{array}$ & 5.365 & 5.138 & 4.715 & 0.281 & 0.848 & No & 0.259 & No & 0.564 & No \\
\hline Turn Bay & 2.491 & 2.392 & 2.274 & 0.564 & 0.877 & No & 0.533 & No & 0.831 & No \\
\hline
\end{tabular}

\section{Lack of Fixations on Pedestrians}

When assessing pedestrian-vehicle conflicts during permissive left-turn operations, it is important to determine if drivers fail to scan for the presence of pedestrians in or adjacent to the crosswalk. The second statistical analysis of the AOI fixation data was focused on proportion of drivers who did or did not fixate on the pedestrians and pedestrian AOI.

$\boldsymbol{H}_{0}:$ There is no difference in the proportion of drivers who fixate on pedestrians or areas where pedestrians would likely be present during permitted left-turn maneuvers at signalized intersections operating the FYA.

Individual driver fixation behavior was examined to determine if failures to fixate while searching for pedestrians took place. As shown in Table 2, at all levels of pedestrian activity a measurable portion of subjects did not fixate on pedestrians or likely pedestrian AOI.

Table 2. Driver fixations on pedestrians

\begin{tabular}{|c|c|c|c|c|c|}
\hline Ped Cases & Total & \multicolumn{2}{|c|}{ Did Not Fixate } & \multicolumn{2}{|c|}{ Fixated } \\
\hline Towards & 152 & 10 & $7 \%$ & 142 & $92 \%$ \\
\hline Away & 150 & 6 & $4 \%$ & 144 & $95 \%$ \\
\hline Both & 309 & 16 & $5 \%$ & 293 & $89 \%$ \\
\hline None & 158 & 62 & $39 \%$ & 96 & $65 \%$ \\
\hline
\end{tabular}

The data show that for the levels of pedestrian activity considered, drivers failed to fixate on pedestrians in the conflicting crosswalk for $4 \%$ to $7 \%$ of the intersection scenarios tested. Comparisons of the proportions between each pedestrian case were made using the proportion test conducted in the R statistical software $(\mathrm{R}, 2012)$. Results are presented in Table 3. 
Table 3. Results of proportions test of pedestrian AOI

\begin{tabular}{|c|c|c|c|}
\hline Comparisons & Difference & $\mathbf{9 5 \%}$ CI & p-value \\
\hline Toward vs Away & $2.6 \%$ & $(-8.3 \%, 3.1 \%)$ & 0.457 \\
\hline Both vs Toward & $1.4 \%$ & $(-6.5 \%, 3.7 \%)$ & 0.690 \\
\hline Both vs Away & $1.2 \%$ & $(-0.3 \%, 5.7 \%)$ & 0.748 \\
\hline None vs Toward & $32.6 \%$ & $(23.4 \%, 41.9 \%)$ & $<0.001$ \\
\hline None vs Away & $35.2 \%$ & $(26.3 \%, 44.1 \%)$ & $<0.001$ \\
\hline None vs Both & $34.1 \%$ & $(25.6 \%, 42.5 \%)$ & $<0.001$ \\
\hline
\end{tabular}

In cases where pedestrians were present, there was no evidence that the two proportions were significantly different for each of the three comparisons $(\alpha=0.05)$. Although no significant differences were found, there is still $4-7 \%$ of the left-turn movements where the drivers "did not look" at pedestrians. This has clear safety impacts for the safe operation of PPLT.

At the intersections that did not have a pedestrian in the conflicting crosswalk, the fixations in the general direction of the pedestrian area were recorded. There were a high number of subjects that did not fixate on these areas where pedestrians could be expected compared to the number of subjects that failed to fixate on pedestrians when they were present. These differences were significant ( $\mathrm{p}$-values $<0.001$ ) for each comparison involving the None pedestrian case.

\section{CONCLUSION}

When in the crosswalk at intersections without protected left-turn phasing, pedestrians are particularly at risk from left-turning vehicles. Though legally required to yield to opposing through vehicles and pedestrians until an acceptable gap is present, it is not uncommon for drivers to fail to observe pedestrians.

This research found statistically significant differences ( $p$-value $<0.001)$ in the average total fixation duration (ATFD) on crossing pedestrians between when a single pedestrian was walking toward the driver and when a total of four pedestrians (two in each direction) were crossing. Additionally, statistically significant differences were found in the ATFD on crossing pedestrians between a single pedestrian crossing away from the driver and from four pedestrians (two in each direction) crossing ( $\mathrm{p}$-value $<0.001)$. These results suggest that when more pedestrians are present in the cross walk, drivers spend more time fixating on those pedestrians.

The results of this experiment also support the notion that when drivers are making a permissive left-turn some drivers fail to search areas where pedestrians are likely to be present. In the study, $7 \%$ of the subjects failed to fixate on pedestrians walking toward their vehicle, $4 \%$ failed when pedestrians were walking away from the subject's vehicle, and 5\% failed to fixate on pedestrians walking in both directions. These percentages suggest that these specific subjects focus on other variables at the intersections and fail to focus on the most vulnerable road users, the pedestrians. In cases where there were no pedestrians present, fixations in the direction of pedestrian areas were collected. $39 \%$ of all subjects failed to fixate on these areas for any potential crossing or queued pedestrians. These results suggest that pedestrian conflicts associated with permissive turns at the FYA could be related to drivers not actively searching for pedestrians, rather than 
"looking and not seeing" or "cognitive distraction". It is important to note that only driver fixations were analyzed. The amount of visual information acquired from saccades or peripheral vision was not considered.

This research has provided insight to driver glance behavior during permissive left-turn maneuvers controlled by FYAs in a driving simulator. The study results have some limitations and clear suggestions for future work. As this research was conducted on a university campus, there was an over-representation of younger drivers. A larger, more diverse sample size could result in more transferrable and applicable findings. Further analyses could be performed. Using the robust eye glance data, fixation sequence (what areas of interest do drivers look at first, second, third, etc.) could be analyzed in more detail. The pedestrian position within the crosswalk when the left-turn is initiated could be analyzed, as well as the acceleration and deceleration comparisons of position data from the simulator when presented with the different variables. Finally, driver behaviors in the simulator could be validated against field data.

\section{ACKNOWLEDGMENTS}

This project was funded by the Oregon Transportation Research and Education Consortium (OTREC) a USDOT University Transportation Center with in-kind matching fund from Washington County, Oregon. Stacy Shetler and Ed Anderson (Washington County) and Shaun Quayle (Kittelson and Associates, Inc.) have contributed oversight and input for the research.

\section{REFERENCES}

Brehmer, C. L., Kacir, K. C., Noyce, D. A., and Manser, M. P. (2003). "Evaluation of Traffic Signal Displays for Protected-Permissive Left Turn Control.” NCHRP Report 493.

Federal Highway Administration. (2009). "Manual on Uniform Traffic Control Devices.” U.S. Department of Transportation.

Knodler, M. A., Noyce, D. A., Kacir, K. C., and Brehmer, C. L. (2001). "Driver Understanding of the Green Ball and Flashing Yellow Arrow Permitted Indications: A Driving Simulator Experiment." ITE, Chicago, Illinois.

Knodler, M. A., and David A. Noyce (2005). "Tracking driver eye movements at permissive leftturns." In Proceedings of the Third International Driving Symposium on Human Factors in Driver Assessment, Training and Vehicle Design, pp. 134-142.

Lord, D., Smiley, A., and Haroun, A. (1998). "Pedestrian Accidents with Left-Turning Traffic at Signalized Intersections: Characteristics, Human Factors and Unconsidered Issues." 77th Annual Transportation Research Board Meeting, Washington, DC.

National Safety Council. (2010). "Understanding the distracted brain: Why driving while using hands-free cell phones is risky behavior." [White paper].

Oregon Department of Transportation (2006). "Traffic Signal Policy and Guidelines.”

Oregon State University (2011). Driving Simulator. Retrieved from Driving and Bicycling Research Lab: http://cce.oregonstate.edu/research/drivingsimulator/DrivingSim.php

R Core Team (2012). R: A language and environment for statistical computing. R Foundation for Statistical Computing, Vienna, Austria. URL http:/www.R-project.org/. 